\section{Research Square}

Preprints are preliminary reports that have not undergone peer review.

They should not be considered conclusive, used to inform clinical practice, or referenced by the media as validated information.

\title{
Development and Design of a Nursing Intervention Following a Theory and Evidence-Based Approach to Promote Maternal Sensitivity and Preterm Infant Neurodevelopment in the NICU
}

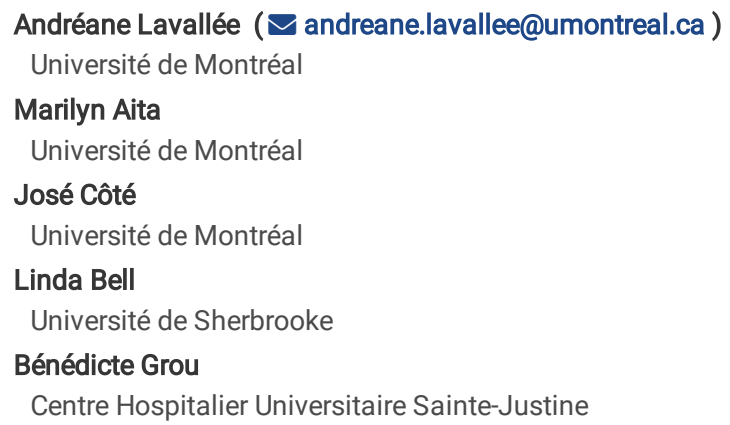




\section{Abstract}

Background: Maternal sensitivity is an important predictor of long-term mother-infant attachment and infant development. Considering the behavioral specificities of preterm infants that may impede the development of maternal sensitivity, it is essential to promote these outcomes soon after a preterm birth. A systematic review showed that current evidence on the effectiveness of parent-infant intervention promoting parental sensitivity in the neonatal intensive care unit (NICU) is of low to very low quality. The aim of this project was to develop and design a novel nursing intervention to enhance maternal sensitivity and preterm infant neurodevelopment in the NICU.

Methods: The Medical Research Council's guidance to develop and evaluate complex health interventions, that is an evidence and theory-based approach, was used for this study. Thus, based on the MRC framework, three main steps were conducted: 1- Identifying existing empirical evidence; 2- Identifying and developing theory; 3- Modeling processes and outcomes.

Results: We developed a guided participation intervention for mothers to participate in their preterm infant's care and positioning (GP_Posit). GP_Posit is based upon the Attachment theory, the Guided participation theory as well as the Synactive theory of development.

Conclusion: This novel intervention is being tested in a pilot randomized controlled trial (NCT03677752).

\section{Background}

Maternal sensitivity is a primary predictor of long-term mother-infant attachment (1). It is defined as the mother's ability to recognize, interpret and respond in an appropriate and timely manner to her infant's behavioral cues (2). In a literature review synthesizing studies using modern neuroimaging techniques to study the parental brain, infant vocalizations and facial expressions were identified as specific cues that trigger regions of the brain to select appropriate caregiving behaviours (3). In fact, maternal sensitivity is a dyadic component of the mother-infant relationship that not only depends upon the mother's ability to detect and interpret infant cues but also on the infant's ability to demonstrate clear cues (4). Thus, being born preterm is an infant factor that may affect parental sensitivity as preterm infants use interaction cues that may be difficult to understand (5). For this reason, current evidence points out that preterm infants may be more susceptible to low-sensitive parenting (6) and require more sensitivity from their mother (7).

Systematic reviews have shown that mothers of preterm infants are as sensitive as those of term infants (7) and that mother-preterm infant dyads are not at greater risk of developing an insecure attachment (8). Nevertheless, it appears there is no consensus regarding the latter statement as attachment is still reported to be less secure in preterm infants compared to term infants between the ages of 12 and 36 months (9). Moreover, early quality of caregiving and mother-infant relationship have systematically been identified as significant predictors of the preterm infant development (10-15). In fact, there is a weak but significant correlation between maternal sensitivity and preterm infant development even after controlling for breastfeeding (16). In a group of 134 infants, neurodevelopment was significantly better in preterm infants whose mothers were qualified as sensitive (17). More specifically, higher levels of maternal sensitivity have been identified as significant predictors of better reading and performances in mathematics at ages seven and eight in children born preterm $(6,13)$. Higher levels of maternal sensitivity also significantly predict larger gray matter volumes and head circumference in preterm infants at the age of eight (18). In light of these results, maternal sensitivity seems to be an important factor having a direct effect upon preterm infants' short-term cognitive and brain development.

Regarding long-term neurodevelopment, impairments are still reported in those children that were born preterm. For example, a recent meta-analysis outlined that children born preterm, compared to children born at term, have significant deficits in mathematics and reading until at least 18 years of age (19). Interestingly, this sample included infants born as late as in 2018, confirming that those deficits remain even in preterm infants who received modern neonatal care (19). Even adults born preterm still report having social difficulties (20) and score significantly lower at neuropsychological tests (21). Thus, preterm infant long-term neurodevelopment is still a contemporary concern. Moreover, considering that early maternal sensitivity has a positive impact upon the preterm infant development, interventions promoting maternal sensitivity during Neonatal Intensive Care Unit (NICU) hospitalization seem necessary. In fact, evidence shows that early interventions implemented during NICU hospitalization enhancing parenting in mothers of preterm infants may act as a leverage for plasticity of the preterm infant's brain to enhance neurodevelopmental outcomes (22).

Our systematic review (23) evaluating the effectiveness of parent-infant interventions in the NICU on sensitivity concluded that these interventions, compared to standard care, did not enhance short-term maternal sensitivity, that being when the preterm infant is at term equivalent age (standardized mean difference [SMD] $0.11 ; 95 \%$ confidence interval $[\mathrm{Cl}]-0.18$ to $0.40 ; \mathrm{p}=0.46 ; \mathrm{I}^{2}=52 \%$ ). Similar results were found at up to 6 months of corrected age (CA) (SMD $0.27 ; 95 \% \mathrm{Cl}$ -0.05 to $\left.0.60 ; p=0.10 ; 1^{2}=60 \%\right)$ and after 6 months of $C A\left(S M D 0.17 ; 95 \% \mathrm{Cl}-0.07\right.$ to $\left.0.41 ; p=0.17 ; I^{2}=8 \%\right)$. Results were the same for preterm infant neurodevelopment at term equivalent age (SMD $0.08 ; 95 \% \mathrm{Cl}-0.19$ to $0.35 ; \mathrm{p}=0.55)$ and after 6 months $\mathrm{CA}(\mathrm{SMD}-0.08 ; 95 \% \mathrm{Cl}-0.61$ to $0.45 ; \mathrm{p}=0.77)$. However, it is important to consider that these results are based on low to very low quality of evidence. In other words, these results may not entirely be due to the ineffectiveness of the interventions, but rather to implementation failure (i.e., intervention not delivered as planned to all participants, dose of the intervention insufficient, contamination between study groups). Qualitative studies and literature reviews have highlighted that nurses have a central role in guiding mothers to develop their relationship with their hospitalized preterm infant $(24,25)$ and for the successful implementation of interventions. It is also recognized by parents that nurses have a key role in facilitating parenting in the NICU (26). In light of these results, it appears important to develop novel theory and evidence-based nursing interventions to add to this body of knowledge. Interventions that were in fact based upon a solid theoretical understanding (27) have shown promising results on maternal sensitivity $(28,29)$ and preterm infant neurodevelopment $(30,31)$. Therefore, the aim was to develop a novel nursing intervention to enhance maternal sensitivity and preterm infant neurodevelopment in the NICU.

\section{Methods}


The novel nursing intervention was developed following a theory and evidence-based approach (32) and more specifically using the Medical Research Council (MRC) Framework for developing and evaluating interventions $(33,34)$. The MRC framework was selected because it offers guidance to develop interventions with a well-founded theoretical understanding to reasonably expect a positive effect on selected outcomes (32, 34). Based on the MRC framework, three main steps were conducted: 1- Identifying existing empirical evidence; 2-Identifying and developing theory; 3 - Modeling processes and outcomes. For the reporting of this intervention development processes in a detailed and exhaustive manner, the Criteria for Reporting the Development and Evaluation of Complex Interventions in healthcare (CReDECl) revised guidelines proposed by Mohler, Kopke (35) for interventions in healthcare were followed. These reporting guidelines included four steps:

1. Description of the appropriate underlying empirical and theoretical basis for the intervention. We started by outlining specific theories as well as available empirical evidence which serve as the basis of the intervention to be developed.

2. Description of the intervention components. In this second step, we identified and described the intervention components, as well as reasons for selecting these components, their characteristics and their specific aim. For the purpose of this description, we defined intervention components as active ingredients that are selected for their potential to induce change on maternal sensitivity and preterm infant neurodevelopment.

3. Illustration of intended interactions between components. In step 3 we clearly illustrated the mechanisms of action and mediators that explain the link between the intervention components and the expected outcomes.

4. Description of contextual characteristics. Finally, we described micro-level context conditions that are relevant for intervention modeling.

After, we present the intervention structure as per the Better reporting of interventions: template for intervention description and replication (TIDieR) by Hoffmann, Glasziou (36). The intervention structure includes the name of the intervention, the materials, the procedures, the provider(s), the modes of delivery, where, when and how much (frequency, duration and dose) as well as possibilities for tailoring the intervention.

\section{Intervention Development Steps}

\subsection{Step 1 - Theories and empirical evidence}

This novel and multifaceted intervention integrates knowledge from experimental studies (empirical evidence) as well as three theories, those being the Attachment Theory $(2,37,38)$, the Synactive Theory of Development (39) and the Guided Participation (GP) theory (40). Each theory contributes to the theoretical foundations of the novel intervention as the attachment theory offers a comprehensive understanding of the mother-infant relationship; the Synactive Theory of Development contributes to the conceptualization of the infant's behavior and development as being influenced by its environment; and the GP theory was identified following our analysis of the empirical evidence to describe the nursing role in the promotion of the parent-infant relationship.

\subsubsection{Attachment Theory}

The attachment theory was originally introduced by John Bowlby (37). Bowlby's ideas originated from animal naturalistic observations that he applied to human infants and their mothers. Bowlby first postulated that attachment encompassed a set of intrinsic behaviors in infants that aimed at maintaining proximity with the mother (37). Before the infant has gained mobility and can demonstrate approaching behaviors, that is behaviors where the infant reaches proximity with his/her mother, he/she will first demonstrate signaling behaviors such as crying, smiling and babbling, that normally bring the mother to the infant (37). Additionally, Bowlby suggested that the mother-infant relationship acts as the base for infant development (38). Our modern conception of the attachment theory is also influenced by Mary Ainsworth, Blehar (2)'s work. Ainsworth later worked on the mother-infant relationship where she was able, following observational studies of human infants and their mothers $(41,42)$, to define different patterns of attachment and components of the mother-infant relationship (2). In fact, Ainsworth introduced the concept of sensitivity where she observed that infants of highly sensitive mothers were more likely to have a secure attachment (41) and a more harmonious mother-infant relationship (43). Highly sensitive mothers are attuned to their infant's cues, respond promptly and appropriately, and understand the meaning of their most subtle signals (2). Infants who have a mother who responds to their needs in such a contingent way feel secure to develop an attachment and to explore their environment (2). The attachment theory oriented the aim of the intervention as it was designed to enhance maternal sensitivity during the first months of the infant's life, such as during NICU hospitalization.

\subsubsection{Synactive Theory of Development}

The Synactive Theory of Development was introduced by Als (39) to allow an understanding of each infant's individuality. Infant's organism develops according to five subsystems: 1) autonomic system, 2) motor system, 3) state-organizational system, 4) attention and interaction system, and 5) selfregulatory system (39). Each of these five subsystems is in interaction with each other and with the environment in order to reach and maintain a state of stability in the infant's organism (39). In fact, even preterm infants have the capacity to interact with their social environment, and this interaction is essential for an optimal development as sensitive interaction partners may help preterm infants to reach a state of stability (39). Infants communicate using cues that are classified in two categories: stress and stability cues. Stress cues translate a state of instability and call for interventions that may help the infant regain a stability state. On the other hand, stability cues call for minimal handling to maintain this state. The main behavioral stress and stability cues, which are most recognizable for parents, are presented in Table 1. In other words, the Synactive Theory of Development stresses the importance for parents and nurses to continuously read infant's behavioral cues as they have the ability of communicating their needs to their environment. 
Table 1

Main stress and stability behavioral cues $^{1}$

\begin{tabular}{|c|c|c|}
\hline Sub-system & Stress & Stability \\
\hline \multirow[t]{4}{*}{ Autonomic } & Hiccuping & \multirow[t]{2}{*}{ Smooth respiration } \\
\hline & Sneezing & \\
\hline & Yawning & \multirow[t]{2}{*}{ Stable color } \\
\hline & Coughing & \\
\hline \multirow[t]{5}{*}{ Motor } & Flaccidity of the trunk and/or extremities & Hand and/or foot clasping \\
\hline & Hyperextension of arms and/or legs and/or trunk & Hand-to-mouth \\
\hline & Finger splays & Grasping \\
\hline & Facial grimacing & Handholding \\
\hline & Tongue extensions & Sucking \\
\hline \multirow[t]{3}{*}{ State } & Crying & Consolability \\
\hline & Irritability & Clear sleep state \\
\hline & Staring & Focused alertness \\
\hline
\end{tabular}

\subsubsection{Guided participation theory}

Based on the empirical literature review on the components and composition of the interventions (see Table 2), we identified GP as being the most frequent mode of delivery of parent-infant interventions to enhance maternal sensitivity (45-51). GP is based upon the GP theory, specific on parenting, which has its origins from the Experiential Learning theory (40). Experiential learning is based upon pragmatist philosophers including mainly Dewey, Lewin and Piaget's ideas (52). Among the pragmatist philosophers, Dewey's strong epistemological foundation is, to its simplest expression, the conception of knowing and doing as being indissociable concepts (53). Dewey gave particular importance to experience that being situations where individuals are subject to the requirements of the environment and plan and adapt their actions according to these environmental conditions (54). Thus, the GP theory integrates these postulates to support the idea that mothers may gain their parental role and develop their relationship with their preterm infant while experiencing caregiving activities (40). Caregiving activities are a set of activities relevant to five main categories: 1) being with the baby, 2) knowing the baby as a person, 3) giving care to the baby, 4) communicating and engaging with others about infant and parental needs, and 5) problem-solving/decision-making/learning (40). Thus, GP is defined as the dyadic process where a novice (mother) engages in a relationship with an expert (nurse), where the former brings the latter to participate in caregiving activities using guidance, over a period of time $(40,55)$. Guidance is more than coaching as it aims to achieve a meaningful goal, that being to bring the mother to acquire an autonomous caregiving practice for her preterm infant (40). Ultimately, mothers develop their relationship with their infant through this caregiving practice (55). The GP theory strongly oriented the identification of main intervention components presented in Sect. 3.2 . 
Table 2

Components and composition of interventions

\begin{tabular}{|c|c|c|c|c|c|c|c|}
\hline References & $\begin{array}{l}\text { Intervention } \\
\text { aims }\end{array}$ & $\begin{array}{l}\text { Intervention } \\
\text { description }\end{array}$ & $\begin{array}{l}\text { How much? } \\
\text { - Number } \\
\text { sessions } \\
\text { - Length of } \\
\text { sessions }\end{array}$ & When? & Modes of delivery & $\begin{array}{l}\text { Professional } \\
\text { delivering } \\
\text { intervention }\end{array}$ & $\begin{array}{l}\text { Parental } \\
\text { role }\end{array}$ \\
\hline $\begin{array}{l}\text { White-Traut } \\
\text { and Nelson } \\
(56)\end{array}$ & $\begin{array}{l}\text { Directly } \\
\text { modifying the } \\
\text { interaction } \\
\text { between mother } \\
\text { and infant via } \\
\text { maternally } \\
\text { administered } \\
\text { intervention. }\end{array}$ & $\begin{array}{l}\text { RISS (Rice Infant } \\
\text { Sensorimotor } \\
\text { Stimulation } \\
\text { Technique): mothers } \\
\text { provided tactile } \\
\text { stimulation (touch or } \\
\text { massage), vestibular } \\
\text { stimulation (rocking), } \\
\text { auditive and visual } \\
\text { stimulation (eye-to-eye } \\
\text { contact). }\end{array}$ & $\begin{array}{l}\text { - Mothers } \\
\text { administered } \\
\text { RISS protocol } \\
\text { four times } \\
\text {-15 minutes }\end{array}$ & $\begin{array}{l}\text { First three } \\
\text { days after } \\
\text { birth. }\end{array}$ & $\begin{array}{l}\text { Verbal } \\
\text { instructions, } \\
\text { pictures that } \\
\text { illustrated the } \\
\text { technique, and } \\
\text { demonstration of } \\
\text { the technique on a } \\
\text { doll, were used for } \\
\text { teaching the RISS. }\end{array}$ & First author (nurse). & $\begin{array}{l}\text { Passive } \\
+ \text { Active }\end{array}$ \\
\hline $\begin{array}{l}\text { Meyer, Coll } \\
(45)\end{array}$ & $\begin{array}{l}\text { Address the } \\
\text { needs of } \\
\text { parents and } \\
\text { their high-risk } \\
\text { infant and } \\
\text { improve } \\
\text { parenting and } \\
\text { family factors } \\
\text { likely to affect } \\
\text { infant } \\
\text { development. }\end{array}$ & $\begin{array}{l}\text { Individualized } \\
\text { intervention based on } \\
\text { initial interview to } \\
\text { identify parent's needs } \\
\text { on four domains: } \\
\text { o NBAS evaluation + } \\
\text { strategies thought to } \\
\text { parents to support } \\
\text { development; } \\
\text { o Family organisation; } \\
\text { o Modification of care } \\
\text { environment + guided } \\
\text { interaction; } \\
\text { o Care after discharge. }\end{array}$ & $\begin{array}{l}\text { - Number of } \\
\text { sessions } \\
\text { depended on } \\
\text { family needs } \\
\text { and duration } \\
\text { of } \\
\text { hospitalisation } \\
\text { - } 1 \mathrm{hr} \text { to } \\
1 \mathrm{hr} 30 \mathrm{~min} \\
\text { sessions }\end{array}$ & $\begin{array}{l}\text { Started when } \\
\text { infant health } \\
\text { was stable } \\
\text { and finished } \\
\text { before } \\
\text { discharge ( } 2 \text { to } \\
8 \text { weeks). }\end{array}$ & $\begin{array}{l}\text { Individualized } \\
\text { family-based } \\
\text { intervention. }\end{array}$ & $\begin{array}{l}\text { Pediatrician, nurse } \\
\text { and physical } \\
\text { therapist. }\end{array}$ & $\begin{array}{l}\text { Passive } \\
+ \text { Active }\end{array}$ \\
\hline $\begin{array}{l}\text { Browne and } \\
\text { Talmi (59) }\end{array}$ & $\begin{array}{l}\text { Increase } \\
\text { knowledge and } \\
\text { contingent } \\
\text { mother infant } \\
\text { interaction and } \\
\text { decrease } \\
\text { parental stress. }\end{array}$ & $\begin{array}{l}\text { Individualized } \\
\text { demonstration of } \\
\text { preterm infant's } \\
\text { reflexes, motor } \\
\text { capacities and sleep- } \\
\text { wake cycles using the } \\
\text { APIB. } \\
\text { Then, mothers were } \\
\text { instructed to interact } \\
\text { with their infant using } \\
\text { the Mother's } \\
\text { Assessment of the } \\
\text { Behavior of her Infant } \\
\text { (MABI). }\end{array}$ & $\begin{array}{l}\cdot \text { One session } \\
\cdot 45 \text { minutes }\end{array}$ & $\begin{array}{l}\text { From NICU } \\
\text { admission to } \\
\text { the week } \\
\text { before } \\
\text { discharge. }\end{array}$ & Individual session. & Not reported. & Active \\
\hline $\begin{array}{l}\text { Melnyk, } \\
\text { Feinstein } \\
\text { (63) }\end{array}$ & $\begin{array}{l}\text { Strengthen } \\
\text { parents' } \\
\text { knowledge and } \\
\text { beliefs about } \\
\text { their preterm } \\
\text { infants and their } \\
\text { own parenting } \\
\text { role and remove } \\
\text { barriers that } \\
\text { would inhibit } \\
\text { them from } \\
\text { participating in } \\
\text { their infants' } \\
\text { care and } \\
\text { interacting with } \\
\text { them in a } \\
\text { developmentally } \\
\text { sensitive } \\
\text { manner. }\end{array}$ & $\begin{array}{l}\text { COPE - Information } \\
\text { given on 1) the } \\
\text { appearance and } \\
\text { behavioral } \\
\text { characteristics of } \\
\text { premature infants } \\
\text { (infant-behavior } \\
\text { information) and how } \\
\text { parents can } \\
\text { participate in their } \\
\text { infants' care, meet } \\
\text { their infants' needs, } \\
\text { enhance quality of } \\
\text { interaction with their } \\
\text { infant, and facilitate } \\
\text { their infant's } \\
\text { development (parent- } \\
\text { role information) and } \\
\text { 2) activities that } \\
\text { assist parents in } \\
\text { implementing the } \\
\text { experimental } \\
\text { information. }\end{array}$ & $\begin{array}{l}\text { - Content was } \\
\text { given to } \\
\text { parents in four } \\
\text { phases } \\
\text { - Duration not } \\
\text { applicable }\end{array}$ & $\begin{array}{l}\text { From first } \\
\text { week after } \\
\text { birth to first } \\
\text { week post } \\
\text { discharge. }\end{array}$ & $\begin{array}{l}\text { Educational- } \\
\text { behavioral } \\
\text { intervention } \\
\text { through audio and } \\
\text { written } \\
\text { information given } \\
\text { to parents. }\end{array}$ & Not applicable. & Passive \\
\hline
\end{tabular}




\begin{tabular}{|c|c|c|c|c|c|c|c|}
\hline References & $\begin{array}{l}\text { Intervention } \\
\text { aims }\end{array}$ & $\begin{array}{l}\text { Intervention } \\
\text { description }\end{array}$ & $\begin{array}{l}\text { How much? } \\
\text { - Number } \\
\text { sessions } \\
\text { - Length of } \\
\text { sessions }\end{array}$ & When? & Modes of delivery & $\begin{array}{l}\text { Professional } \\
\text { delivering } \\
\text { intervention }\end{array}$ & $\begin{array}{l}\text { Parental } \\
\text { role }\end{array}$ \\
\hline $\begin{array}{l}\text { Glazebrook, } \\
\text { Marlow (60) }\end{array}$ & $\begin{array}{l}\text { Enhance } \\
\text { parents' } \\
\text { observations of } \\
\text { their baby and } \\
\text { sensitivity to } \\
\text { cues through a } \\
\text { series of } \\
\text { activities which } \\
\text { follow the } \\
\text { progression of } \\
\text { care from } \\
\text { incubator to } \\
\text { home. }\end{array}$ & $\begin{array}{l}\text { Parent Baby } \\
\text { Interaction Program } \\
\text { (PBIP): Tactile, } \\
\text { discussion, verbal, } \\
\text { observation and } \\
\text { developmental care } \\
\text { activities. }\end{array}$ & $\begin{array}{l}\text { - Weekly } \\
\text { sessions } \\
\cdot 1-\mathrm{hr}\end{array}$ & $\begin{array}{l}\text { From NICU } \\
\text { admission to } \\
\text { discharge. } \\
\text { Option to } \\
\text { continue six } \\
\text { weeks after } \\
\text { discharge. }\end{array}$ & $\begin{array}{l}\text { Individual } \\
\text { sessions. }\end{array}$ & Neonatal nurse. & Active \\
\hline $\begin{array}{l}\text { Chiu and } \\
\text { Anderson } \\
(61)\end{array}$ & $\begin{array}{l}\text { Enhance } \\
\text { mother-preterm } \\
\text { infant } \\
\text { interaction. }\end{array}$ & $\begin{array}{l}\text { Preterm infants } \\
\text { placed in skin-to-skin } \\
\text { contact (SSC) after } \\
\text { birth. SSC was } \\
\text { encouraged as long } \\
\text { as possible and as } \\
\text { frequently as possible. } \\
\text { Guidance provided to } \\
\text { recognize hunger } \\
\text { cues. }\end{array}$ & $\begin{array}{l}\text { - One session } \\
\text { + guidance } \\
\text { during SSC } \\
\text { - Not reported }\end{array}$ & $\begin{array}{l}\text { From birth to } \\
\text { NICU } \\
\text { discharge. }\end{array}$ & $\begin{array}{l}\text { At birth mothers } \\
\text { are encouraged to } \\
\text { begin SSC as early, } \\
\text { as often, and for } \\
\text { as long as } \\
\text { possible. }\end{array}$ & Nurse. & Active \\
\hline $\begin{array}{l}\text { Newnham, } \\
\text { Milgrom (46) }\end{array}$ & $\begin{array}{l}\text { Enhance the } \\
\text { quality of } \\
\text { mother-infant } \\
\text { interaction by } \\
\text { teaching the } \\
\text { mothers of low- } \\
\text { birth-weight } \\
\text { infants to be } \\
\text { more sensitive } \\
\text { and responsive } \\
\text { to their babies' } \\
\text { physiological } \\
\text { and social cues. }\end{array}$ & $\begin{array}{l}\text { Modified Mother- } \\
\text { Infant Transaction } \\
\text { Program (MITP): } \\
\text { psychoeducational } \\
\text { intervention on five } \\
\text { main topics: 1) } \\
\text { becoming acquainted, } \\
\text { 2) Recognizing infant } \\
\text { disorganisation/stress } \\
\text { and availability and } \\
\text { then applying those } \\
\text { principles during care } \\
\text { and play, 3) bath } \\
\text { session, where } \\
\text { mothers (who bathed } \\
\text { their infants) were } \\
\text { helped to recognize, } \\
\text { and appropriately } \\
\text { respond to infant } \\
\text { disorganisation and } \\
\text { stress, 4) regulate } \\
\text { infant responses } \\
\text { through play in home } \\
\text { setting, and 5) } \\
\text { Recognizing } \\
\text { temperamental } \\
\text { characteristics in } \\
\text { infants and parenting } \\
\text { to different } \\
\text { temperaments }\end{array}$ & $\begin{array}{l}\text { - Nine } \\
\text { sessions } \\
\cdot 30 \text { min. to } \\
\text { 1- hr }\end{array}$ & $\begin{array}{l}\text { Weeks before } \\
\text { discharge to } 3 \\
\text { months CA. }\end{array}$ & $\begin{array}{l}\text { Verbal instruction, } \\
\text { infant observation, } \\
\text { practical } \\
\text { experience in } \\
\text { handling infants } \\
\text { and modeling, as } \\
\text { well as written } \\
\text { materials. }\end{array}$ & $\begin{array}{l}\text { First author } \\
\text { (psychologist). }\end{array}$ & $\begin{array}{l}\text { Passive } \\
+ \text { Active }\end{array}$ \\
\hline
\end{tabular}




\begin{tabular}{|c|c|c|c|c|c|c|c|}
\hline References & $\begin{array}{l}\text { Intervention } \\
\text { aims }\end{array}$ & $\begin{array}{l}\text { Intervention } \\
\text { description }\end{array}$ & $\begin{array}{l}\text { How much? } \\
\text { - Number } \\
\text { sessions } \\
\text { - Length of } \\
\text { sessions }\end{array}$ & When? & Modes of delivery & $\begin{array}{l}\text { Professional } \\
\text { delivering } \\
\text { intervention }\end{array}$ & $\begin{array}{l}\text { Parental } \\
\text { role }\end{array}$ \\
\hline $\begin{array}{l}\text { Teti, Black } \\
(57)\end{array}$ & $\begin{array}{l}\text { Promote } \\
\text { parents' } \\
\text { awareness of } \\
\text { infant bodily } \\
\text { and social cues } \\
\text { and parents' } \\
\text { sensitivity and } \\
\text { confidence in } \\
\text { handling their } \\
\text { infants. } \\
\text { Provide parents } \\
\text { with } \\
\text { information, via } \\
\text { instruction and } \\
\text { demonstration, } \\
\text { about } \\
\text { premature } \\
\text { infants' } \\
\text { capacities for } \\
\text { interacting with } \\
\text { the world, how } \\
\text { to recognize } \\
\text { and respond } \\
\text { appropriately to } \\
\text { infant cues, and } \\
\text { the role of } \\
\text { parent-infant } \\
\text { interaction in } \\
\text { optimizing } \\
\text { infant } \\
\text { development. }\end{array}$ & $\begin{array}{l}\text { Parentally } \\
\text { administered infant } \\
\text { massage designed to } \\
\text { promote infant } \\
\text { development and, in } \\
\text { this case, parental } \\
\text { knowledge of subtle } \\
\text { infant cues and } \\
\text { feeling of intimacy } \\
\text { toward their infants. } \\
\text { Parent-focused } \\
\text { psychoeducational } \\
\text { intervention designed } \\
\text { to foster parents' } \\
\text { knowledge about } \\
\text { preterm infants' } \\
\text { capabilities and needs } \\
\text { and how best to read, } \\
\text { respond to, and } \\
\text { facilitate infant social } \\
\text { behavior. }\end{array}$ & $\begin{array}{l}\cdot \text { Eight } \\
\text { sessions } \\
\cdot 1-\mathrm{hr}\end{array}$ & $\begin{array}{l}20 \text {-week } \\
\text { intervention } \\
\text { from NICU to } \\
\text { post- } \\
\text { discharge. }\end{array}$ & Not reported. & Not reported. & $\begin{array}{l}\text { Passive } \\
+ \text { Active }\end{array}$ \\
\hline $\begin{array}{l}\text { Ravn, Smith } \\
(47)\end{array}$ & $\begin{array}{l}\text { Help parents } \\
\text { appreciate their } \\
\text { infant's unique } \\
\text { characteristics, } \\
\text { temperament, } \\
\text { and } \\
\text { developmental } \\
\text { potential, make } \\
\text { the parents } \\
\text { more sensitive } \\
\text { and responsive } \\
\text { to their infants' } \\
\text { physiological } \\
\text { and social cues, } \\
\text { particularly } \\
\text { those that } \\
\text { signal stimulus } \\
\text { overload. }\end{array}$ & $\begin{array}{l}\text { MITP: Interaction } \\
\text { guidance focusing on } \\
\text { teaching the parents } \\
\text { to understand the } \\
\text { individuality of an } \\
\text { atypical child, to } \\
\text { establish a good } \\
\text { pattern of interaction } \\
\text { and to encourage the } \\
\text { parents to enjoy their } \\
\text { infants. }\end{array}$ & $\begin{array}{l}\cdot \text { Eleven } \\
\text { sessions } \\
\cdot 1-\mathrm{hr}\end{array}$ & $\begin{array}{l}\text { Last week } \\
\text { before } \\
\text { discharge to } \\
\text { 3rd month } \\
\text { after } \\
\text { discharge. }\end{array}$ & $\begin{array}{l}\text { Individualized } \\
\text { interaction } \\
\text { guidance. }\end{array}$ & Neonatal nurses. & Active \\
\hline $\begin{array}{l}\text { Zelkowitz, } \\
\text { Feeley (58) }\end{array}$ & $\begin{array}{l}\text { Reduce } \\
\text { maternal } \\
\text { anxiety and } \\
\text { enhance } \\
\text { maternal } \\
\text { sensitivity by } \\
\text { intervening at } \\
\text { the level of both } \\
\text { maternal } \\
\text { distress and } \\
\text { maternal } \\
\text { interactive } \\
\text { behavior to } \\
\text { promote a better } \\
\text { parenting } \\
\text { environment } \\
\text { and thereby } \\
\text { optimize the } \\
\text { child's cognitive } \\
\text { and social } \\
\text { development. }\end{array}$ & $\begin{array}{l}\text { CUES: educational } \\
\text { intervention for } \\
\text { mothers to } \\
\text { o recognize signs of } \\
\text { their anxiety/distress; } \\
\text { o use various } \\
\text { strategies to alleviate } \\
\text { their distress; } \\
\text { o read their infant's } \\
\text { communication cues; } \\
\text { o respond sensitively } \\
\text { to their infant's cues } \\
\text { and distress. }\end{array}$ & $\begin{array}{l}\cdot \text { Six sessions } \\
\text { •1-hr }\end{array}$ & $\begin{array}{l}\text { Starting } ~ 33 \\
\text { days after } \\
\text { birth until } 6-8 \\
\text { weeks of CA. }\end{array}$ & $\begin{array}{l}\text { Individual } \\
\text { sessions of } \\
\text { teaching and one } \\
\text { individual of video } \\
\text { interaction } \\
\text { guidance. }\end{array}$ & $\begin{array}{l}\text { Nurse or } \\
\text { psychologist. }\end{array}$ & $\begin{array}{l}\text { Passive } \\
+ \text { Active }\end{array}$ \\
\hline
\end{tabular}




\begin{tabular}{|c|c|c|c|c|c|c|c|}
\hline References & $\begin{array}{l}\text { Intervention } \\
\text { aims }\end{array}$ & $\begin{array}{l}\text { Intervention } \\
\text { description }\end{array}$ & $\begin{array}{l}\text { How much? } \\
\text { - Number } \\
\text { sessions } \\
\text { - Length of } \\
\text { sessions }\end{array}$ & When? & Modes of delivery & $\begin{array}{l}\text { Professional } \\
\text { delivering } \\
\text { intervention }\end{array}$ & $\begin{array}{l}\text { Parental } \\
\text { role }\end{array}$ \\
\hline $\begin{array}{l}\text { Milgrom, } \\
\text { Newnham } \\
(64)\end{array}$ & $\begin{array}{l}\text { Enhance the } \\
\text { quality of } \\
\text { mother-infant } \\
\text { interaction by } \\
\text { teaching the } \\
\text { mothers of low- } \\
\text { birth-weight } \\
\text { infants to be } \\
\text { more sensitive } \\
\text { and responsive } \\
\text { to their babies' } \\
\text { physiological } \\
\text { and social cues. }\end{array}$ & $\begin{array}{l}\text { Enhanced MITP } \\
\text { (PremieStart } \\
\text { program). } \\
\text { Mothers trained to } \\
\text { increase their } \\
\text { sensitivity in } \\
\text { recognizing signs of } \\
\text { infant stress including } \\
\text { "shut-down" } \\
\text { mechanisms, alert- } \\
\text { available behavior, } \\
\text { facial expressions, } \\
\text { quality of motor } \\
\text { behaviors, posture } \\
\text { and muscle tone; how } \\
\text { to provide graded } \\
\text { stimulation; and how } \\
\text { to avoid } \\
\text { overwhelming infants; } \\
\text { focus on touch, } \\
\text { movement and } \\
\text { massage; SSC; multi- } \\
\text { sensory stimulation; } \\
\text { debriefing and } \\
\text { normalizing parental } \\
\text { feelings; challenging } \\
\text { dysfunctional } \\
\text { thoughts, and parental } \\
\text { diary keeping. }\end{array}$ & $\begin{array}{l}\text { - Nine weekly } \\
\text { sessions and } \\
\text { one session } \\
\text { post-discharge } \\
\text {-1-hr }\end{array}$ & $\begin{array}{l}\text { From first } \\
\text { week after } \\
\text { birth to first } \\
\text { week post } \\
\text { discharge. }\end{array}$ & $\begin{array}{l}\text { Individual } \\
\text { psychoeducational } \\
\text { intervention. }\end{array}$ & Psychologists. & Passive \\
\hline $\begin{array}{l}\text { Borghini, } \\
\text { Habersaat } \\
(49)\end{array}$ & $\begin{array}{l}\text { Enhance the } \\
\text { quality of } \\
\text { parental } \\
\text { caregiving as a } \\
\text { support to the } \\
\text { infant's global } \\
\text { development by } \\
\text { improving } \\
\text { parents' } \\
\text { observation and } \\
\text { understanding } \\
\text { of the specific } \\
\text { competencies } \\
\text { of their preterm } \\
\text { infant } \\
\text { (particularly } \\
\text { interactional } \\
\text { competencies) } \\
\text { and promote } \\
\text { parents' } \\
\text { sensitivity and } \\
\text { responsiveness } \\
\text { towards their } \\
\text { infant's } \\
\text { behavioral } \\
\text { characteristics. }\end{array}$ & $\begin{array}{l}\text { At } 33 \text { weeks of GA: } \\
\text { joint observation of } \\
\text { infant's reactions to } \\
\text { various stimuli during } \\
\text { standard care } \\
\text { procedures. } \\
\text { At } 4 \text { months CA: 10- } \\
\text { min mother-infant free } \\
\text { play videotaped } \\
\text { followed by video } \\
\text { interaction guidance. }\end{array}$ & $\begin{array}{l}\cdot \text { Four } \\
\text { sessions } \\
\cdot 30-60 \\
\text { minutes }\end{array}$ & $\begin{array}{l}\text { First session } \\
\text { at } 33 \text { weeks of } \\
\text { GA. Three } \\
\text { sessions one- } \\
\text { week apart } \\
\text { during 4th } \\
\text { month CA. }\end{array}$ & $\begin{array}{l}\text { Individual } \\
\text { sessions of } \\
\text { interaction } \\
\text { guidance and } \\
\text { video interaction } \\
\text { guidance. }\end{array}$ & Nurse and therapist. & Active \\
\hline
\end{tabular}




\begin{tabular}{|c|c|c|c|c|c|c|c|}
\hline References & $\begin{array}{l}\text { Intervention } \\
\text { aims }\end{array}$ & $\begin{array}{l}\text { Intervention } \\
\text { description }\end{array}$ & $\begin{array}{l}\text { How much? } \\
\text { - Number } \\
\text { sessions } \\
\text { - Length of } \\
\text { sessions }\end{array}$ & When? & Modes of delivery & $\begin{array}{l}\text { Professional } \\
\text { delivering } \\
\text { intervention }\end{array}$ & $\begin{array}{l}\text { Parental } \\
\text { role }\end{array}$ \\
\hline $\begin{array}{l}\text { Hane, Myers } \\
(28)\end{array}$ & $\begin{array}{l}\text { Establish an } \\
\text { emotional } \\
\text { connection and } \\
\text { a Calming Cycle } \\
\text { routine between } \\
\text { the mother and } \\
\text { her premature } \\
\text { infant. }\end{array}$ & $\begin{array}{l}\text { Family Nurture } \\
\text { Intervention (FNI): } \\
\text { mothers are involved } \\
\text { in calming } \\
\text { interventions which is } \\
\text { facilitated by a nurture } \\
\text { specialist. } \\
\text { o Scent cloth } \\
\text { exchange; } \\
\text { o Vocal soothing and } \\
\text { emotion expression; } \\
\text { o Eye contact; } \\
\text { o Skin-to-skin and } \\
\text { clothed holding. }\end{array}$ & $\begin{array}{l}\cdot \text { Average of } \\
3.5 \\
\text { sessions/week } \\
\cdot \sim 6 \mathrm{hr} / \text { week }\end{array}$ & $\begin{array}{l}\text { From NICU } \\
\text { admission to } \\
\text { discharge } \\
\text { depending on } \\
\text { availabilities } \\
\text { of mothers. }\end{array}$ & $\begin{array}{l}\text { Individual } \\
\text { sessions of guided } \\
\text { participation. }\end{array}$ & $\begin{array}{l}\text { Nurture specialists } \\
\text { (former nurses). }\end{array}$ & Active \\
\hline $\begin{array}{l}\text { Hoffenkamp, } \\
\text { Tooten (50) }\end{array}$ & $\begin{array}{l}\text { Facilitate } \\
\text { parental } \\
\text { bonding, to } \\
\text { enhance the } \\
\text { quality of } \\
\text { parental } \\
\text { interactive } \\
\text { behavior, and to } \\
\text { promote } \\
\text { parental well- } \\
\text { being using } \\
\text { edited video } \\
\text { recordings of } \\
\text { parent-infant } \\
\text { interactions. }\end{array}$ & $\begin{array}{l}\text { Parent-infant } \\
\text { interaction videotaped } \\
\text { during daily moments } \\
\text { of caregiving such at } \\
\text { bathing, changing and } \\
\text { feeding. Video is then } \\
\text { reviewed and } \\
\text { discussed with the } \\
\text { parents. }\end{array}$ & $\begin{array}{l}\text { - Three } \\
\text { sessions } \\
\text { - Duration not } \\
\text { reported }\end{array}$ & $\begin{array}{l}\text { During first } \\
\text { week after } \\
\text { birth. }\end{array}$ & $\begin{array}{l}\text { Individual } \\
\text { sessions of video } \\
\text { interaction } \\
\text { guidance. }\end{array}$ & $\begin{array}{l}\text { Trained nurses and } \\
\text { pedagogic workers. }\end{array}$ & Active \\
\hline $\begin{array}{l}\text { Evans, Boyd } \\
(65)\end{array}$ & $\begin{array}{l}\text { Prevent severe } \\
\text { emotional, } \\
\text { behavioral and } \\
\text { developmental } \\
\text { problems in } \\
\text { both children } \\
\text { and } \\
\text { adolescents. }\end{array}$ & $\begin{array}{l}\text { Triple } \mathbf{P} \text { is an } \\
\text { educational } \\
\text { intervention covering } \\
\text { four main topics: } 1 \text { ) } \\
\text { survival skills, 2) } \\
\text { partner support, } 3 \text { ) } \\
\text { positive parenting, } \\
\text { and } 4 \text { ) responding to } \\
\text { your baby. }\end{array}$ & $\begin{array}{l}\text { - Four } \\
\text { sessions } \\
\cdot 2-\text { hr } \\
\text { - Four post- } \\
\text { discharge } \\
\text { phone calls } \\
\text {-30-minutes }\end{array}$ & $\begin{array}{l}\text { From NICU } \\
\text { hospitalisation } \\
\text { to } 12 \text { months } \\
\text { CA. }\end{array}$ & Not reported. & Trained facilitators. & Passive \\
\hline $\begin{array}{l}\text { Sahlen } \\
\text { Helmer, } \\
\text { Birberg } \\
\text { Thornberg } \\
\text { (62) }\end{array}$ & $\begin{array}{l}\text { Increase time } \\
\text { spent in SSC to } \\
\text { improve mother- } \\
\text { infant } \\
\text { interaction. }\end{array}$ & $\begin{array}{l}\text { Continuous skin-to- } \\
\text { skin contacts between } \\
\text { mother and infant. }\end{array}$ & $\begin{array}{l}\text { - Continuously } \\
\text { (>20-hr/day) }\end{array}$ & $\begin{array}{l}\text { First seven } \\
\text { days of life. }\end{array}$ & Not applicable. & Not applicable. & Active \\
\hline $\begin{array}{l}\text { Twohig, } \\
\text { Segurado } \\
(51)\end{array}$ & Not reported. & $\begin{array}{l}\text { Reflective interview, } \\
\text { observation of infant } \\
\text { cues and video } \\
\text { interaction guidance. }\end{array}$ & $\begin{array}{l}\cdot \text { Three } \\
\text { sessions } \\
\text { • 1-hr to 1.5-hr }\end{array}$ & Not reported. & $\begin{array}{l}\text { Individual } \\
\text { sessions. }\end{array}$ & $\begin{array}{l}\text { First author } \\
\text { (clinician/researcher) }\end{array}$ & Active \\
\hline
\end{tabular}

\subsubsection{Empirical evidence}

We conducted a systematic review evaluating the effectiveness of parent-preterm infant interventions, conducted in the NICU, on parental sensitivity (23). Based on this systematic review, a secondary thorough analysis of the components and composition of the interventions ( $n=18)$ was conducted (see Table 2). This content analysis has allowed the identification of the main intervention components as described in Sect. 3.2.

\subsection{Step 2 - Components of the intervention}

Based on the theories and empirical evidence, two main intervention components were identified: 1- an educational component, and 2- an active-participatory component. These two components are interconnected and both essential. The intervention developed is hence multifaceted as it includes both passive and active components, the former being educational activities where mothers receive information and the latter where mothers actively participate to caregiving activities. In fact, both passive and active components seem to have their importance in parent-infant interventions with a predominance of a combination of the two components $(45,46,56-58)$, or an active component alone $(28,47-51,59-62)$. As outlined in Table 3 , the educational component encompasses different topics, and the active-participatory component relates to maternal participation to various caregiving activities of their preterm infant with direct guidance from a nurse. The rationale for selecting these two components is further explained in Sect. 3.2.1. and 3.2.2. 
Table 3

GP_Posit intervention components

\begin{tabular}{ll} 
Educational \\
Topics & Stress and stability behavioral cues of preterm infants \\
\cline { 2 - 2 } & Supine, lateral and prone positioning \\
\hline-
\end{tabular}

Active-Participatory

Caregiving activities

Diapering

Positioning (supine, lateral and prone)

Bottle-feeding and/or breastfeeding (optional)

\subsubsection{Educational component}

The educational component includes teaching to mothers aspects about stress and stability behavioral cues of preterm infants, which is supported by both theory $(2,39)$ and empirical evidence $(46,47,49,51,57-59,63-65)$. In fact, if mothers are expected to detect and interpret their infant's cues $(2)$, and considering that preterm infants communicate with cues that are difficult to understand (5), it becomes evident that those specific cues should first be thought to mothers before they may interpret and respond to them.

Additionally, in the studies identified in the systematic review, many parent-infant interventions were centered around teaching mothers about care activities that may enhance their preterm infant's neurodevelopment. Some interventions aimed at teaching mothers about sensorimotor stimulation (56), multisensorial stimulation $(28,48,64)$, massage (57), developmental care $(60)$, or skin-to-skin $(61,62)$.

In this intervention, mothers are being taught how to position their preterm infant in their incubator or crib. First, positioning is a central part of preterm infant's care while in the NICU intended to improve their neuromotor development (66). Maternal participation to positioning has been evaluated in one randomized controlled trial (RCT) (67) where, compared to standard care, preterm infants who received the motor intervention from their mothers had a significantly better motor performance at term equivalent age (68). Mothers in this study also noted that it empowered them to become competent in providing care and enhanced their feeling of attachment to their preterm infant (69). Based on this evidence, teaching mothers how to position their preterm infant while in the NICU was selected.

\subsubsection{Active-participatory component}

In addition to the educational component, the emphasis of the intervention is on the active-participatory component (40). In fact, in $50 \%$ of the parent-infant interventions from the systematic review, parents provided care to their preterm infant, and in $75 \%$ of those, parents were given specific guidance to do so (4551). Thus, parents participate in caregiving activities of their preterm infant, while being guided by a nurse.

In this intervention, mothers will actively participate to caregiving activities with their preterm infants while being guided by a nurse. It is of importance to note that the caregiving activities are only the context provided to mothers so they can learn how to interact with their preterm infant. Thus, while providing care to their infant, guidance is given to mothers so they can learn to detect, interpret and respond to their infant's behavioral stress and stability cues. In fact, for preterm infants, caregiving activities are recognized as being stressful $(70,71)$, so care should be provided in accordance with their behavioral cues (72). In other words, the nurse's role during the sessions is to provide guidance to mothers by encouraging, praising, and supporting them in recognizing behavioral cues when they did or did not recognize or respond to a cue. As stated in Table 3, caregiving activities include diapering, positioning and feeding (optional). When providing care to preterm infants in the NICU, diapering is usually the first manipulation done, followed by repositioning. Thus, throughout the intervention sessions, mothers will progressively participate to their infant's diapering, then supine, lateral and prone positioning. This progression will be based both on the mothers' ability and level of confidence as well as the nurse's judgment. Nevertheless, the aim is to focus on the mother-infant communication while doing the caregiving activity and not necessarily go through every caregiving activity during the sessions. However, if both the mother and the nurse feel that the mother easily recognizes, interprets and responds to most behavioral cues of her infant while doing a specific caregiving activity, they may move onto the next caregiving activity. For infants nearing home discharge and who are learning to feed orally, the caregiving activity could be adapted to bottle or breastfeeding if mothers are already comfortable with positioning.

\subsection{Step 3 - Interactions between components}

Figure 1 exposes the intricate links between intervention components, mechanisms of action, mediators as well as expected outcomes of GP_Posit, based on theories and empirical evidence. These links are described thereafter.

\subsubsection{Links between intervention components and mechanisms of action}

The educational component about behavioral cues should increase mother's knowledge about their infant's cues as well as their belief about their competence in recognizing these cues $(5,39,73,74)$. Mothers of preterm infants verbalize the need to be educated about these cues (75). As for the positioning educational component, this should contribute to maternal ease in the guided participation to diapering and positioning. Maternal active participation to care through GP will trigger five mechanisms of action. First, in interventions where touching the infant is involved, the feeling of physical proximity within the dyad in increased (76). GP as well as maternal active participation to care increase the feeling of gaining confidence in maternal role and lowering maternal stress $(77,78)$. Moreover, having mothers participate to their preterm infant's positioning should promote optimal positioning practices for the preterm infant throughout the NICU stay. Finally, as the emphasis in GP is primarily to support mothers in communicating with their preterm infant, this entails that they give importance to recognizing in addition to interpreting the stress and stability cues. Respecting preterm infant cues while providing care helps them to selfregulate and keep their stress to a minimal level (39). Also, appropriate positioning of the preterm infant in the NICU allows them to improve autoregulation $(79,80)$

\section{$22 ?$ I inke hatiasoan machanisms of action, mediators and outcomes


Mothers being more knowledgeable about their preterm infant's behavioral cues should allow them to be better prepared to detect and interpret these cues which is central to maternal sensitivity (2) and to reduce stress and anxiety (63). Physical proximity between the mother and her infant is the base of the attachment theory and also contributes to maternal sensitivity (37).

Mother-infant interventions in the NICU where parental participation is promoted have reduced maternal anxiety (81). On the opposite, physical proximity is limited, maternal anxiety increases (82), so promoting physical proximity in the intervention should reduce maternal anxiety.

Parental role adjustment is one of the most important sources of stress for mothers in the NICU $(83,84)$. Having mothers participate in their infant's care has been identified as an intervention promoting the mother's confidence in her maternal role (84) and thus will reduce maternal stress and anxiety (85).

Preterm infant positioning is an integral part of preterm infant care in the NICU (86) for its benefits on neuromotor development $(80,87,88)$. Stress in the NICU is detrimental for the preterm infant's neurodevelopment (89), so promoting preterm infants' positioning and reducing their stress is expected to enhance their neurodevelopment.

\subsubsection{Links between mediators and outcomes}

A concept analysis of maternal sensitivity identified maternal anxiety as a negative affecting factor to maternal sensitivity (90). Lowering maternal stress has systematically been identified as favorable to improve maternal sensitivity $(5,90,91)$. In other words, reducing maternal stress and anxiety should increase their sensitivity.

\subsubsection{Links between the outcome of maternal sensitivity and infant neurodevelopment}

Many studies have linked an increase in maternal sensitivity to preterm infants' neurodevelopment. For example, enhanced maternal sensitivity predicts larger gray matter volume and head circumference (18), improved mental development (14), more consistent and symmetric cortical thickness across brain hemispheres (92), improved cognitive performances $(6,13,16)$ and improved cerebral white matter micro-structural development (93).

\subsection{Step 4: Contextual characteristics}

The intervention will be implemented in a NICU designed with single-family rooms (SFR). This is to take into consideration since it has been demonstrated that SFR ensure more privacy, promote family-centered care, and provide a more favorable environment to build trust between nurses and parents (94, 95).

\section{Intervention Structure}

\subsection{Materials}

This intervention mainly relies upon the relationship developed between the nurse and the mother which requires no material. However, to support the educational component of the intervention, an informative booklet and web-based modules are used to support the nurse's teaching.

Firstly, the informative booklet, developed by the first author (AL), contains pictures of various stress and stability cues so the nurse can use those pictures during the educational component of the intervention. Mothers can refer to it between intervention sessions. The booklet also contains pictures of appropriately positioned preterm infants so they can also have a reference if they want to participate in their preterm infant's positioning in between intervention sessions.

Secondly, the nurse also has access to web-based modules developed by a multidisciplinary team that provides written information as well as pictures and videos adapted for parents of preterm infants that demonstrate appropriate techniques of positioning in the NICU (96). The web-based modules were previously pilot tested, and results showed that parents were satisfied, and that the positioning module was most liked by parents (97). The advantage of the online modules is that mothers may refer to it at any time.

\subsection{Name of the intervention}

This intervention was named GP_Posit because it mainly focuses on Guided Participation of mothers to the Positioning of their preterm infant.

\subsection{Procedures}

The structure and procedures of the GP_Posit intervention sessions are presented in Table 4. 
Table 4

GP_Posit intervention structure

\begin{tabular}{|lll|}
\hline Sessions & Intervention passive and active components & Content of session \\
\hline Session 1 & Education & Preterm infant development \\
& Participation & Stress and stability behavioral cues \\
Session 2 & Education & Diapering \\
\cline { 2 - 3 } & Participation & Supine positioning \\
Session 3 & Education & Diapering + supine positioning \\
\cline { 2 - 4 } & Participation & Lateral positioning \\
\hline Session 4 & Education & Diapering + lateral positioning \\
\cline { 2 - 3 } & Participation & Prone positioning \\
\hline Session $5+$ & Participation & Diapering + prone positioning \\
& & Diapering + positioning \\
& & Feeding (optional) \\
\hline
\end{tabular}

\subsection{Provided by who}

GP_Posit intervention is meant to be administered by trained neonatal registered nurses as they have the expertise to work with preterm infants and their mothers. In fact, in our systematic review conducted to support this intervention, $\geq 55 \%$ of parent-infant interventions were provided by nurses. However, for GP_Posit, nurses should be able to educate mothers about the behavioral cues of their preterm infants and appropriate positioning techniques. So, if this training is not already provided in the NICU where the intervention is to be implemented, the intervention nurses should receive appropriate training on those topics. Additionally, neonatal nurses should be trained in GP, about what GP entails, their role as expert nurses, as well as how to administer GP. Most importantly, the same nurse should always meet with the same mothers throughout the sessions as GP is based on the mother-nurse relationship developed over time (40).

\subsection{Modes of delivery}

GP_Posit is delivered through individual sessions, between the nurse and the mother. This choice was based on the fact that $\geq 60 \%$ of the parent-infant intervention were provided through individual sessions. Also, GP entails that sessions should be individualized (40).

\subsection{Where}

GP_Posit will be implemented in a level III NICU of a university hospital center. Sessions are provided at each infant's bedside, in SFRs to ensure privacy and a calmer environment (94).

\subsection{When and how much}

In our systematic review, 11 out of the 18 identified interventions were mainly conducted during NICU hospitalization and finished, at the latest, one week after discharge $(28,45,50,51,56,59-64)$. Moreover, the average number of sessions that were delivered was 5 sessions throughout the intervention, varying from one to 11 sessions. As for the length of each session, the average reported in interventions from the systematic review was of 62 minutes per sessions, varying from 15 minutes to 2 hours.

Thus, GP_Posit is meant to be implemented as soon as possible after birth and should be performed until the infant reaches 35 weeks of gestational age (GA) or until discharge at home. The intervention will be offered to mothers of preterm infants born at 28 weeks of GA or more, as preterm infants start showing behavioral cues around that age (98). The number of sessions will depend on the age of the infant at birth and age at discharge. For example, mothers of infants born at 31 weeks of GA would participate in four sessions and mothers of infants born at 28 weeks of GA, seven sessions. The sessions should take place weekly, and each has a duration of 30 to $45 \mathrm{~min}$ or more depending on time needed for the completion of care. If possible, these sessions should be timed with each preterm infant's care plan in the NICU and should be clustered with other care as suggested (72), so that she/he is not awakened uniquely for the intervention purposes. Also, for pragmatic considerations, the schedule for the individual sessions should be determined with the mother, according to her availabilities.

\subsection{Tailoring}

Considering the importance of individualizing interventions for preterm infants (44), the care used to contextualize the mother-infant interaction could be tailored based on the mother's needs, abilities and level of confidence. For example, the content of each session is pre-planned (see Table 4), but nurses are free to adapt this content depending on specific infant and maternal needs at time of the session.

\section{Discussion}

In this paper we presented the development process of a nurse-led intervention of guided participation in the NICU designed to enhance maternal sensitivity and preterm infant neurodevelopment. The development of this intervention is novel as it is anchored in a theory and evidence-based approach such as the Loading [MathJax]/jax/output/CommonHTML/jax.js 
MRC framework and brings a unique contribution to the neonatal body of knowledge. The strength of this approach is that it allowed to follow a systematic methodology to develop a thorough understanding of the underpinning processes that predict the effectiveness of the intervention components on selected outcomes. Theories to support GP_Posit were identified for their relevance to optimally understand the nurse's role to contribute to the attachment process between mothers and their hospitalized preterm infant. In addition to grand theories $(2,39,40)$, we were also able to build upon strengths and limitations of previous interventions evaluated in RCTs and identified through our systematic review (23).

\section{Conclusion}

In the NICU, nurses are recognized as being the primary caregivers of preterm infants with parents. GP_Posit is a novel multifaceted nursing intervention aiming at building a stronger partnership between mothers and nurses to guide mothers in gaining faster their maternal role. In other words, based on empirical and theoretical evidence, we expect that mothers participating in GP_Posit will develop a stronger maternal sensitivity and that preterm infants will demonstrate enhanced neurodevelopment. Thus, this intervention has the potential to enhance neonatal nursing care and optimize both mothers' and preterm infants' short- and long-term outcomes. GP_Posit intervention was piloted according to a published protocol to evaluate mother's acceptability and satisfaction with the intervention as well as preliminary effects on maternal sensitivity and preterm infant neurodevelopment (99).

\section{Abbreviations}

CA: corrected age

Cl: confidence interval

CReDECl: Criteria for Reporting the Development and Evaluation of Complex Interventions in healthcare

GA: gestational age

GP_Posit: Guided Participation to care and Positioning and care intervention

MRC: medical research council

NICU: neonatal intensive care unit

RCT: randomized controlled trial

SMD: standardized mean difference

TIDieR: Better reporting of interventions: template for intervention description and replication

\section{Declarations}

\section{Ethics approval and consent to participate}

Not applicable.

\section{Consent for publication}

Not applicable.

\section{Availability of data and materials}

Data sharing is not applicable to this article as no datasets were generated or analysed during the current study.

\section{Competing interests}

The authors declare that they have no competing interests.

\section{Funding}

Not applicable.

\section{Authors' contributions}

AL led intervention development and modeling. MA, JC, LB and BG provided guidance and feedback, read, edited and approved the final manuscript.

\section{Acknowledgements}

AL was supported by a CIHR Fellowship (2018MFE-416125-FAH-CHCH-268206) and the Faculty of Nursing of the Université de Montréal for her doctoral studies. AL also acknowledges fellowships received for her doctoral studies from the Ministère de l'Éducation et de l'Enseignement Supérieur (MEES), Ordre 
des Infirmières et Infirmiers du Québec (OIIQ) and Quebec Network on Nursing Interventions (RRISIQ) (2016-2018), Fonds de Recherche du Québec - Santé (FRQ-S) (2018-2019), the TD funds at the CHU Sainte-Justine (2015-2017) and the Levinschi foundation at the CHU Sainte-Justine (2016-2017; 2019).

\section{References}

1. Deans CL. Maternal sensitivity, its relationship with child outcomes, and interventions that address it: a systematic literature review. Early Child Development and Care. 2018;190(2):252-75.

2. Ainsworth MD, Blehar MC, Waters E, Wall SN. Patterns of Attachment: A Psychological Study of the Strange Situation: Erlbaum, Hillside; 1978.

3. Young KS, Parsons CE, Stein A, Vuust P, Craske MG, Kringelbach ML. The neural basis of responsive caregiving behaviour: Investigating temporal dynamics within the parental brain. Behav Brain Res. 2017;325(Pt B):105-16.

4. Oxford M, Findlay D. NCAST Caregiver / Parent-Child interaction Feeding Manual. 2 ed. Seattle: Parent-chil relationship programs University od Washington School of Nursing; 2015.

5. Neuhauser A. Predictors of maternal sensitivity in at-risk families. Early Child Development and Care. 2016;188(2):126-42.

6. Jaekel J, Pluess M, Belsky J, Wolke D. Effects of maternal sensitivity on low birth weight children's academic achievement: a test of differential susceptibility versus diathesis stress. J Child Psychol Psychiatry. 2015;56(6):693-701.

7. Bilgin A, Wolke D. Maternal Sensitivity in Parenting Preterm Children: A Meta-analysis. Pediatrics. 2015;136(1):e177-93.

8. Korja R, Latva R, Lehtonen L. The effects of preterm birth on mother-infant interaction and attachment during the infant's first two years. Acta Obstet Gynecol Scand. 2012;91(2):164-73.

9. Ruiz N, Piskernik B, Witting A, Fuiko R, Ahnert L. Parent-child attachment in children born preterm and at term: A multigroup analysis. PLoS One. 2018;13(8):e0202972.

10. Poehlmann J, Hane A, Burnson C, Maleck S, Hamburger E, Shah PE. Preterm infants who are prone to distress: differential effects of parenting on 36month behavioral and cognitive outcomes. J Child Psychol Psychiatry. 2012;53(10):1018-25.

11. Grunberg VA, Geller PA, Bonacquisti A, Patterson CA. NICU infant health severity and family outcomes: a systematic review of assessments and findings in psychosocial research. J Perinatol. 2019;39(2):156-72.

12. Wright N, Hill J, Sharp H, Pickles A. Maternal sensitivity to distress, attachment and the development of callous-unemotional traits in young children. J Child Psychol Psychiatry. 2018;59(7):790-800.

13. Treyvaud K, Doyle LW, Lee KJ, Ure A, Inder TE, Hunt RW, et al. Parenting behavior at 2 years predicts school-age performance at 7 years in very preterm children. J Child Psychol Psychiatry. 2016;57(7):814-21.

14. Treyvaud K, Anderson VA, Howard K, Bear M, Hunt RW, Doyle LW, et al. Parenting behavior is associated with the early neurobehavioral development of very preterm children. Pediatrics. 2009;123(2):555-61 7p.

15. Stein A, Malmberg LE, Leach P, Barnes J, Sylva K, Team F. The influence of different forms of early childcare on children's emotional and behavioural development at school entry. Child Care Health Dev. 2013;39(5):676-87.

16. Banerjee N. Are maternal depression, breastfeeding, matenal alcohol intake, and infant biological vulnerability, effect modifiers of confounders of the maternal sensitivity-cognitive development association? 2018.

17. Neri E, Agostini F, Baldoni F, Facondini E, Biasini A, Monti F. Preterm infant development, maternal distress and sensitivity: The influence of severity of birth weight. Early Hum Dev. 2017;106-107:19-24.

18. Kok R, Thijssen S, Bakermans-Kranenburg MJ, Jaddoe VW, Verhulst FC, White T, et al. Normal variation in early parental sensitivity predicts child structural brain development. J Am Acad Child Adolesc Psychiatry. 2015;54(10):824-31 e1.

19. McBryde M, Fitzallen GC, Liley HG, Taylor HG, Bora S. Academic Outcomes of School-Aged Children Born Preterm: A Systematic Review and Metaanalysis. JAMA. 2020;3(4):e202027.

20. Pyhala R, Wolford E, Kautiainen H, Andersson S, Bartmann P, Baumann N, et al. Self-Reported Mental Health Problems Among Adults Born Preterm: A Meta-analysis. Pediatrics. 2017;139(4).

21. O'Reilly H, Johnson S, Ni Y, Wolke D, Marlow N. Neuropsychological Outcomes at 19 Years of Age Following Extremely Preterm Birth. Pediatrics. 2020;145(2).

22. DeMaster D, Bick J, Johnson U, Montroy JJ, Landry S, Duncan AF. Nurturing the preterm infant brain: leveraging neuroplasticity to improve neurobehavioral outcomes. Pediatr Res. 2019;85:166-75.

23. Lavallée A, De Clifford-Faugere G, Ballard A, Aita M. Parent-infant interventions to promote parental sensitivity during the NICU hospitalization: Systematic review and meta-analysis. Journal of Early Intervention. accepted manuscript.

24. Fernandez Medina IM, Granero-Molina J, Fernandez-Sola C, Hernandez-Padilla JM, Camacho Avila M, Lopez Rodriguez MDM. Bonding in neonatal intensive care units: Experiences of extremely preterm infants' mothers. Women Birth. 2018;31(4):325-30.

25. Fleck P. Connecting Mothers and Infants in the Neonatal Intensive Care Unit. Newborn Infant Nurs Rev. 2016;16(2):92-6.

26. Reid S, Bredemeyer S, Chiarella M. Integrative Review of Parents' Perspectives of the Nursing Role in Neonatal Family-Centered Care. J Obstet Gynecol Neonatal Nurs. 2019;48(4):408-17.

27. Welch MG, Hofer MA, Brunelli SA, Stark RI, Andrews HF, Austin J, et al. Family nurture intervention (FNI): Methods and treatment protocol of a randomized controlled trial in the NICU. BMC Pediatr. 2012;12(14):e1-17.

Loading [MathJax]/jax/output/CommonHTML/jax.js 
28. Hane A, Myers MM, Hofer MA, Ludwig RJ, Halperin MS, Austin J, et al. Family Nurture Intervention Improves the Quality of Maternal Caregiving in the Neonatal Intensive Care Unit: Evidence from a Randomized Controlled Trial. J Dev Behav Pediatr. 2015;36(3):188-96.

29. Beebe B, Myers MM, Lee SH, Lange A, Ewing J, Rubinchik N, et al. Family nurture intervention for preterm infants facilitates positive mother-infant face-toface engagement at 4 months. Dev Psychol. 2018;54(11):2016-31.

30. Welch MG, Myers MM, Grieve PG, Isler JR, Fifer WP, Sahni R, et al. Electroencephalographic activity of preterm infants is increased by Family Nurture Intervention: a randomized controlled trial in the NICU. Clin Neurophysiol. 2014;125(4):675-84.

31. Welch MG, Stark RI, Grieve PG, Ludwig RJ, Isler JR, Barone JL, et al. Family nurture intervention in preterm infants increases early development of cortical activity and independence of regional power trajectories. Acta Paediatr. 2017;106(12):1952-60.

32. O'Cathain A, Croot L, Sworn K, Duncan E, Rousseau N, Turner K, et al. Taxonomy of approaches to developing interventions to improve health: a systematic methods overview. Pilot \& Feasibility Studies. 2019;5:41.

33. Craig P, Dieppe P, Macintyre S, Michie S, Nazareth I, Petticrew M, et al. Developing and evaluating complex interventions: the new Medical Research Council guidance. BMJ. 2008;337:a1655.

34. Craig P, Dieppe P, Macintyre S, Michie S, Nazareth I, Petticrew M. Developing and evaluating complex interventions: the new Medical Research Council guidance. Int J Nurs Stud. 2013;50(5):587-92.

35. Mohler R, Kopke S, Meyer G. Criteria for Reporting the Development and Evaluation of Complex Interventions in healthcare: revised guideline (CReDECI 2). Trials. 2015;16:204.

36. Hoffmann TC, Glasziou PP, Boutron I, Milne R, Perera R, Moher D, et al. Better reporting of interventions: template for intervention description and replication (TIDieR) checklist and guide. BMJ. 2014;348:g1687.

37. Bowlby J. Attachment and Loss. 2 ed. New York: Basic Books; 1982.

38. Bowlby J. A Secure Base: Parent-Child Attachment and Healthy Human Development USA: Basic Books; 1988.

39. Als H. Toward a Synactive Theory of Development: Promise for the Assessment and Support of Infant individuality. Infant Ment Health J. 1982;3(4):22943.

40. Pridham KF, Limbo R, Schroeder M, Thoyre S, Van Riper M. Guided participation and development of care-giving competencies for families of low birthweight infants. J Adv Nurs. 1998;28(5):948-58.

41. Ainsworth MD. The development of infant-mother interaction among the Ganda. In: Foss BM, editor. Determinants of infant behavior. New York, NY: Wiley; 1963. p. 67-104.

42. Ainsworth MD. Infancy in Uganda: Infant care and the growth of love. Baltimore: Johns Hopkins University Press; 1967.

43. Bell SM, Ainsworth MDS. Infant crying and maternal responsiveness. Child Dev. 1972;43:1171-90.

44. Als H, Lawhon G, Brown E, Gibes-Grossman R, Duffy FH, McAnulty GB, et al. Individualized Behavioral and Environmental Care for the Very Low Birth Weight Preterm Infant at High Risk for Bronchopulmonary Dysplasia : Neonatal Intensive Care Unit Developmental Outcome. Pediatrics. 1986;78(6):112332.

45. Meyer EC, Coll CT, Lester BM, Boukydis CF, McDonough SM, Oh W. Family-based intervention improves maternal psychological well-being and feeding interaction of preterm infants. Pediatrics. 1994;93(2):241-6.

46. Newnham CA, Milgrom J, Skouteris H. Effectiveness of a modified Mother-Infant Transaction Program on outcomes for preterm infants from 3 to 24 months of age. Infant Behavior and Development. 2009;32(1):17-26.

47. Ravn IH, Smith L, Lindemann R, Smeby NA, Kyno NM, Bunch EH, et al. Effect of early intervention on social interaction between mothers and preterm infants at 12 months of age: a randomized controlled trial. Infant Behavior and Development. 2011;34(2):215-25.

48. White-Traut RC, Norr KF, Fabiyi C, Rankin KM, Li Z, Liu L. Mother-infant interaction improves with a developmental intervention for mother-preterm infant dyads. Infant Behavior and Development. 2013;36(4):694-706.

49. Borghini A, Habersaat S, Forcada-Guex M, Nessi J, Pierrehumbert B, Ansermet F, et al. Effects of an early intervention on maternal post-traumatic stress symptoms and the quality of mother-infant interaction: the case of preterm birth. Infant Behav Dev. 2014;37(4):624-31.

50. Hoffenkamp HN, Tooten A, Hall RA, Braeken J, Eliens MP, Vingerhoets AJ, et al. Effectiveness of hospital-based video interaction guidance on parental interactive behavior, bonding, and stress after preterm birth: A randomized controlled trial. J Consult Clin Psychol. 2015;83(2):416-29.

51. Twohig A, Segurado R, McCarthy A, Underdown A, McNicholas F, Molloy EJ. Early intervention to support preterm infant-parent interaction and development: results of a randomised controlled trial on maternal sensitivity, social-emotional development and parental mental health. Arch Dis Child. 2019;103(Suppl 3):A62-A3.

52. Miettinen R. The concept of experiential learning and John Dewey's theory of reflective thought and action. International Journal of Lifelong Education. 2000;19(1):54-72.

53. Talisse RB, Aikin SF. The Pragmatism Reader: From Peirce through the Present. Oxford, UK: Princteton University Press; 2011.

54. Dewey J. Experience and Nature: Read Books Ltd; 2013.

55. Schroeder M, Pridham KF. Development of Relationship Competencies Through Guided Participation for Mothers of Preterm Infants. Journal of Obstetric, Gynecologic \& Neonatal Nursing. 2006;35(3):358-68.

56. White-Traut RC, Nelson MN. Maternally administered tactile, auditory, visual, and vestibular stimulation: relationship to later interactions between mothers and premature infants. Res Nurs Health. 1988;11(1):31-9.

Loading [MathJax]/jax/output/CommonHTML/jax.js 
57. Teti DM, Black MM, Viscardi R, Glass P, O'Connell MA, Baker L, et al. Intervention With African American Premature Infants: Four-Month Results of an Early Intervention Program. Journal of Early Intervention. 2009;31(2):146-66.

58. Zelkowitz P, Feeley N, Shrier I, Stremler R, Westreich R, Dunkley D, et al. The cues and care randomized controlled trial of a neonatal intensive care unit intervention: effects on maternal psychological distress and mother-infant interaction. J Dev Behav Pediatr. 2011;32(8):591-9.

59. Browne JV, Talmi A. Family-based intervention to enhance infant-parent relationships in the neonatal intensive care unit. J Pediatr Psychol. 2005;30(8):667-77.

60. Glazebrook C, Marlow N, Israel C, Croudace T, Johnson S, White IR, et al. Randomised trial of a parenting intervention during neonatal intensive care. Archives of Disease in Childhood Fetal \& Neonatal Edition. 2007;92(6):F438-43.

61. Chiu SH, Anderson GC. Effect of early skin-to-skin contact on mother-preterm infant interaction through 18 months: randomized controlled trial. Int J Nurs Stud. 2009;46(9):1168-80.

62. Sahlen Helmer C, Birberg Thornberg U, Frostell A, Ortenstrand A, Morelius E. A Randomized Trial of Continuous Versus Intermittent Skin-to-Skin Contact After Premature Birth and the Effects on Mother-Infant Interaction. Adv Neonatal Care. 2019.

63. Melnyk BM, Feinstein NF, Alpert-Gillis L, Fairbanks E, Crean HF, Sinkin RA, et al. Reducing premature infants' length of stay and improving parents' mental health outcomes with the Creating Opportunities for Parent Empowerment (COPE) neonatal intensive care unit program: a randomized, controlled trial. Pediatrics. 2006;118(5):e1414-27.

64. Milgrom J, Newnham C, Martin PR, Anderson PJ, Doyle LW, Hunt RW, et al. Early communication in preterm infants following intervention in the NICU. Early Hum Dev. 2013;89(9):755-62.

65. Evans T, Boyd RN, Colditz P, Sanders M, Whittingham K. Mother-Very Preterm Infant Relationship Quality: RCT of Baby Triple P. Journal of Child and Family Studies. 2017;26(1):284-95.

66. Lavallée A, De Clifford-Faugere G, Matte C, Aita M. Effets bénéfiques du positionnement sur le développement du nouveau-né prématuré. Cahiers de la Puéricultrice. 2018;55(316):15-8.

67. Øberg GK, Campbell SK, Girolami GL, Ustad T, Jørgensen L, Kaaresen PI. Study protocol: an early intervention program to improve motor outcome in preterm infants: a randomized controlled trial and a qualitative study of physiotherapy performance and parental experiences. BMC Pediatr. 2012;12(15).

68. Ustad T, Evensen KAI, Campbell SK, Girolami GL, Helbostad J, Jørgensen L, et al. Early Parent-Administered Physical Therapy for Preterm Infants: A Randomized Controlled Trial. Pediatrics. 2016;138(2):e20160271.

69. Øberg GK, Ustad T, Jørgensen L, Kaaresen PI, Labori C, Girolami GL. Parents' perceptions of administering a motor intervention with their preterm infant in the NICU. European Journal of Physiotherapy. 2018;21(3):134-41.

70. Peng NH, Bachman J, Chen CH, Huang LC, Lin HC, Li TC. Energy expenditure in preterm infants during periods of environmental stress in the neonatal intensive care unit. Jpn J Nurs Sci. 2014;11(4):241-7.

71. Pereira FL, Nogueira de Goes Fdos S, Fonseca LM, Scochi CG, Castral TC, Leite AM. Handling of preterm infants in a neonatal intensive care unit. Rev Esc Enferm USP. 2013;47(6):1272-8.

72. Lavallée A, De Clifford-Faugère G, Garcia C, Fernandez Oviedo AN, Héon M, Aita M. PART 2: Practice and research recommendations for quality developmental care in the NICU. J Neonatal Nurs. 2019.

73. Als H. A synactive model of neonatal behavioral organization : framework for the assessment of neurobehavioral development in the premature infant and for support of infants and parents in the neonatal intensive care environment. Phys Occup Ther Geriatr. 1986;6(3-4):3-53.

74. Melnyk BM, Oswalt KL, Sidora-Arcoleo K. Validation and psychometric properties of the neonatal intensive care unit parental beliefs scale. Nurs Res. 2014;63(2):105-15.

75. Lee SN, Long A, Boore J. Taiwanese women's experiences of becoming a mother to a very-low-birth-weight preterm infant: a grounded theory study. Int J Nurs Stud. 2009;46(3):326-36.

76. Feeley N, Genest C, Niela-Vilen H, Charbonneau L, Axelin A. Parents and nurses balancing parent-infant closeness and separation: a qualitative study of NICU nurses' perceptions. BMC Pediatr. 2016;16:134.

77. Cleveland LM. Parenting in the Neonatal Intensive Care Unit. J Obstet Gynecol Neonatal Nurs. 2008;37(6):666-91.

78. Smith VC, Steelfisher GK, Salhi C, Shen LY. Coping with the neonatal intensive care unit experience: parents' strategies and views of staff support. J Perinat Neonatal Nurs. 2012;26(4):343-52.

79. Jarus T, Bart O, Rabinovich G, Sadeh A, Bloch L, Dolfin T, et al. Effects of prone and supine positions on sleep state and stress responses in preterm infants. Infant Behavior and Development. 2011;34(2):257-63.

80. King C, Norton D. Does therapeutic positioning of preterm infants impact upon optimal health outcomes? A literature review. J Neonatal Nurs. 2017.

81. Welch MG, Halperin MS, Austin J, Stark RI, Hofer MA, Hane AA, et al. Depression and anxiety symptoms of mothers of preterm infants are decreased at 4 months corrected age with Family Nurture Intervention in the NICU. Arch Womens Ment Health. 2016;19(1):51-61.

82. Vazquez V, Cong X. Parenting the NICU infant: A meta-ethnographic synthesis. International Journal of Nursing Sciences. 2014;1(3):281-90.

83. Roque ATF, Lasiuk GC, Radunz V, Hegadoren K. Scoping Review of the Mental Health of Parents of Infants in the NICU. J Obstet Gynecol Neonatal Nurs. 2017;46(4):576-87.

84. Govindaswamy P, Laing S, Waters D, Walker K, Spence K, Badawi N. Needs and stressors of parents of term and near-term infants in the NICU: A systematic review with best practice guidelines. Early Hum Dev. 2019;139:104839.

Loading [MathJax]/jax/output/CommonHTML/jax.js 
85. Melnyk BM, Crean HF, Feinstein NF, Fairbanks E, Alpert-Gillis LJ. Testing the theoretical framework of the COPE program for mothers of critically ill children: an integrative model of young children's post-hospital adjustment behaviors. J Pediatr Psychol. 2007;32(4):463-74.

86. Lavallée A, De Clifford-Faugère G, Garcia C, Fernandez Oviedo AN, Héon M, Aita M. Part 1: Narrative overview of developmental care interventions for the preterm newborn. J Neonatal Nurs. 2019;25(1):3-8.

87. Blauw-Hospers $\mathrm{CH}$, de Graaf-Peters VB, Dirks T, Bos AF, Hadders-Algra M. Does early intervention in infants at high risk for a developmental motor disorder improve motor and cognitive development? Neurosci Biobehav Rev. 2007;31(8):1201-12.

88. Sweeney JK, Heriza CB, Blanchard Y, Dusing SC. Neonatal physical therapy. Part II: Practice frameworks and evidence-based practice guidelines. Pediatr Phys Ther. 2010;22(1):2-16.

89. Graven SN, Browne JV. Sensory Development in the Fetus, Neonate, and Infant: Introduction and Overview. Newborn Infant Nurs Rev. 2008;8(4):169-72.

90. Shin H, Park YJ, Ryu H, Seomun GA. Maternal sensitivity: a concept analysis. J Adv Nurs. 2008;64(3):304-14.

91. Booth AT, Macdonald JA, Youssef GJ. Contextual stress and maternal sensitivity: A meta-analytic review of stress associations with the Maternal Behavior Q-Sort in observational studies. Dev Rev. 2018;48:145-77.

92. Frye RE, Malmberg B, Swank P, Smith K, Landry S. Preterm birth and maternal responsiveness during childhood are associated with brain morphology in adolescence. J Int Neuropsychol Soc. 2010;16(5):784-94.

93. Milgrom J, Newnham C, Anderson PJ, Doyle LW, Gemmill AW, Lee K, et al. Early sensitivity training for parents of preterm infants: impact on the developing brain. Pediatr Res. 2010;67(3):330-5.

94. Winner-Stoltz R, Lengerich A, Hench AJ, O'Malley J, Kjelland K, Teal M. Staff Nurse Perceptions of Open-Pod and Single Family Room NICU Designs on Work Environment and Patient Care. Adv Neonatal Care. 2018;18(3):189-98.

95. Doede M, Trinkoff AM. Emotional Work of Neonatal Nurses in a Single-Family Room NICU. J Obstet Gynecol Neonatal Nurs. 2020.

96. Luu TM, Gosselin J, Karsenti T, Côté S, Walker DC, Peckre P, et al. Mieux Agir au Quotidien : Comprendre et soutenir le développement de mon enfant 2015 [Available from: http://developpementenfant.ca/wp/.

97. Luu TM, Xie LF, Peckre P, Cote S, Karsenti T, Walker CD, et al. Web-Based Intervention to Teach Developmentally Supportive Care to Parents of Preterm Infants: Feasibility and Acceptability Study. JMIR Research Protocols. 2017;6(11):e236.

98. Fern D. A neurodevelopmental care guide to positioning \& handling the premature, fragile or sick infant. New York, NY: DF Publishing; 2011.

99. Lavallee A, Aita M, Cote J, Bell L, Luu TM. A guided participation nursing intervention to theraupeutic positioning and care (GP_Posit) for mothers of preterm infants: protocol of a pilot randomized controlled trial. Pilot Feasibility Studies. 2020;6:77.

\section{Figures}

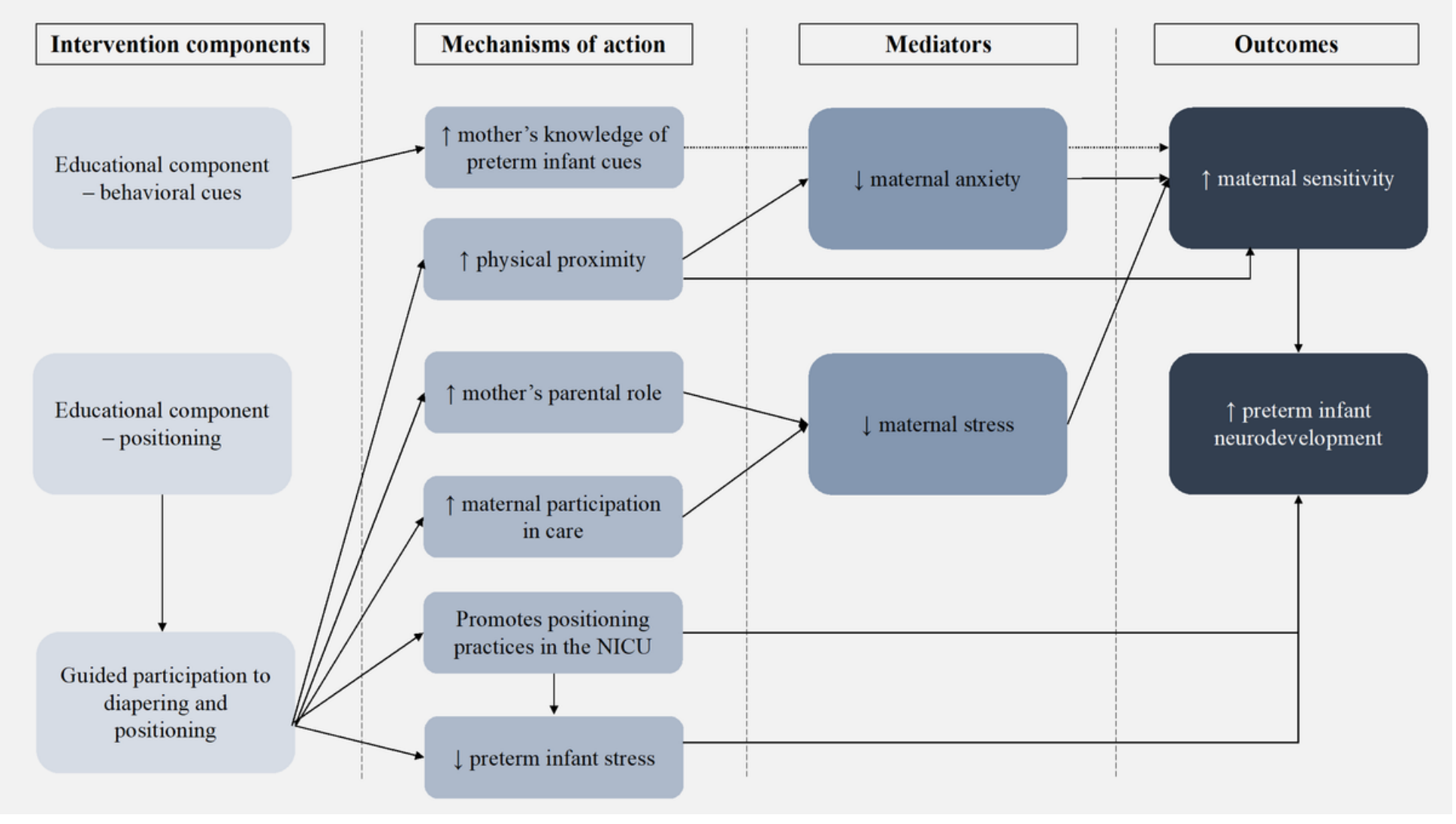

Figure 1

Loading [MathJax]/jax/output/CommonHTML/jax.js

Page $17 / 18$ 
Integration of intervention components, mechanisms of action, mediators and outcomes

Loading [MathJax]/jax/output/CommonHTML/jax.js

Page 18/18 